Disclosure of Interests None declared

DOI: 10.1136/annrheumdis-2019-eular.5830

\section{AB0851 1 OBESITY AND RISK OF FRACTURE: DEPENDS ON THE BODY MASS INDEX AND TYPE OF FRACTURE}

Jose Rosas ${ }^{1}$, Catalina Cano ${ }^{1}$, Ana Pons ${ }^{1}$, Estíbaliz Ivars ${ }^{1}$, José Miguel SenabreGallego ${ }^{1}$, Gregorio Santos Soler ${ }^{1}$, Esteban Salas-Heredia', José Antonio BernalVidal', José Alberto García-Gómez ${ }^{2}$, Xavier Barber ${ }^{3}$, AIRE-MB. ${ }^{1}$ Hospital Marina Baixa, Rheumatology, Villajoyosa (Alicante), Spain; ${ }^{2}$ Elche General University Hospital, Infectious Diseases, Elche (Alicante), Spain; ${ }^{3}$ Miguel Hernández University, Statistics, Elche (Alicante), Spain

Objectives: To know the impact of obesity on the risk of fracture. Methods: Observational study, during 2010-2018, of patients referred for bone densitometry (BMD), to the Bone Densitometry Unit of Rheumatology.

BMD performed by Rheumatology nursing, collect several data (age, gender); osteoporosis (OP) risk factors: age of menopause, smoking and current alcohol intake, hip fracture parents, body mass index (BMI), diseases-drugs that reduce bone mass, fractures, treatment; BMD in lumbar spine (LS), femoral neck (FN); FRAX with BMD. BMI result was as WHO recommendations in $\mathrm{kg} / \mathrm{m}^{2}$ : Normal: $18.5-24.99$; Obesity: $\geq 30$; type I: 30-34.99, type II: 35.39.99, type III: $\geq 40$.

Results: A first BMD in 6.943 postmenopausal women was done. 2.196 $(32 \%)$ had normal BMl, low weight $\left(\mathrm{BMl}<18.5 \mathrm{~kg} / \mathrm{m}^{2}\right) 103$ (1.5\%) patients, overweight (BMI 25-29.99 kg/m²) $2.643(38 \%)$ and obesity $2.001(29 \%)$.

Obesity (BMI $\left.\geq 30 \mathrm{~kg} / \mathrm{m}^{2}\right)$ vs normal $\mathrm{BMI}$, are older $(65 \pm 13$ vs $61 \pm 13$, $\mathrm{p}=0.005)$, lower current smokers $(12 \%$ vs $27 \%) \mathrm{P}<0.0001)$, more treated for OP (37\% vs $29 \%, p=0.002)$, higher percentage of fractures $(44 \%$ vs $29 \%)$, especially distal radius (DR) (30\% vs $11 \%), p<0.001)$ and vertebral (19\% vs $7 \%, p=0.002)$, lower mean $T$ score in LS $(-1.1 \pm 1.5$ vs $1.8 \pm 1.5$, $p<0.0001)$ and $F N(-1.1 \pm 1.5$ vs $-1.6 \pm 1.5, p<0.0001)$ and lower mean FRAX hip fracture with BMD $(1.9 \pm 3.6$ vs $2.4 \pm 3.6, \mathrm{p}<0.0001)$. In table, obesity groups are compared with normal BMI.

Conclusion: In postmenopausal women referred for BMD, obese women vs normal BMI are 1. Older. 2. Overall, with higher percentage of fractures. 3. But, women with $\mathrm{BMI} \geq 40 \mathrm{~kg} / \mathrm{m}^{2}$ do not have a higher overall percentage of fracture, except in DR.

\begin{tabular}{|c|c|c|c|c|c|c|c|}
\hline & $\begin{array}{c}\text { IMC } \\
\text { Normal } \\
18.5- \\
24.9 \\
\text { N: 2.196 }\end{array}$ & $\begin{array}{c}\text { IMC } \\
\text { Obesity I } \\
\text { 30-34.99 } \\
\text { N: 1.403 }\end{array}$ & $\begin{array}{c}p \\
\text { Normal } \\
\text { vs } \\
\text { Obesity I }\end{array}$ & $\begin{array}{c}\text { IMC } \\
\text { obesity II } \\
35-39.99 \\
\text { N: 421 }\end{array}$ & $\begin{array}{c}\mathrm{P} \\
\text { Normal } \\
\text { vs } \\
\text { Obesity II }\end{array}$ & $\begin{array}{c}\text { IMC } \\
\text { Obesity III } \\
\geq 40 \\
\text { N: } 177\end{array}$ & $\begin{array}{c}p \\
\text { Normal } \\
\text { us } \\
\text { Obesity II }\end{array}$ \\
\hline $\begin{array}{l}\text { Age years, } \\
\text { mean (SD) }\end{array}$ & $\begin{array}{c}61.4 \\
(13.1)\end{array}$ & $\begin{array}{l}65.6 \\
(13.1)\end{array}$ & 0.0001 & $\begin{array}{l}65.7 \\
(13.1)\end{array}$ & 0.0001 & $\begin{array}{l}63.5 \\
(13.1)\end{array}$ & 0.04 \\
\hline BMI, mean (SD) & $\begin{array}{l}22.7 \\
(5.2)\end{array}$ & $\begin{array}{l}32.1 \\
\{5.2\}\end{array}$ & 0.0001 & $\begin{array}{l}36.8 \\
\{5.2\}\end{array}$ & 0.0001 & $\begin{array}{l}43.4 \\
\text { (5.2) }\end{array}$ & 0.0001 \\
\hline $\begin{array}{l}\text { Menopause } \\
\text { age, mean (SS) }\end{array}$ & $\begin{array}{l}47.3 \\
(5.5)\end{array}$ & $\begin{array}{l}47.6 \\
\{5.5\}\end{array}$ & 0.11 & $\begin{array}{l}47.8 \\
\{5.4\}\end{array}$ & 0.08 & $\begin{array}{l}48.3 \\
\text { (5.5) }\end{array}$ & 0.02 \\
\hline $\begin{array}{l}\text { Current } \\
\text { smoker, } \mathrm{n}(\%)\end{array}$ & $587(27)$ & $176(13)$ & 0.0001 & $40(10)$ & 0.02 & $19(11)$ & 0.23 \\
\hline $\begin{array}{l}\text { Hip fracture in } \\
\text { mother, } \mathrm{n}(\%)\end{array}$ & $254(12)$ & $144(10)$ & 0.54 & $36(9)$ & 0.59 & $16(9)$ & 0.71 \\
\hline $\begin{array}{l}\begin{array}{l}\text { Current } \\
\text { treatment, n } \\
\text { (\%) }\end{array}\end{array}$ & 645 (29) & $516(37)$ & 0.004 & $147(35)$ & 0.15 & $58(33)$ & 0.52 \\
\hline $\begin{array}{l}\text { Corticosteroids, } \\
n(\%)\end{array}$ & 156 (7) & $94(7)$ & 1 & $32(8)$ & 0.84 & $18(10)$ & 0.64 \\
\hline $\begin{array}{l}\text { Fracture, } n(\%) \\
\text { Colles } \\
\text { Vertebral } \\
\text { Hip } \\
\text { Humerus } \\
\text { Other }\end{array}$ & $\begin{aligned} 645(29) \\
235(11) \\
145(7) \\
66(3) \\
22(1) \\
194(29)\end{aligned}$ & $\begin{array}{c}520(37) \\
160(30) \\
101(19) \\
32(6) \\
53(10) \\
193(36 \%)\end{array}$ & $\begin{array}{l}0.004 \\
0.0001 \\
0.004 \\
0.47 \\
0.17 \\
0.14\end{array}$ & $\begin{array}{c}168(40) \\
58(32) \\
32(18) \\
11(6) \\
14(8) \\
66(36 \%)\end{array}$ & $\begin{array}{c}0.006 \\
0.0001 \\
0.049 \\
0.61 \\
0.28 \\
0.28 \\
\end{array}$ & $\begin{array}{c}60(34) \\
20(32) \\
7(11) \\
4(6) \\
8(12) \\
23(37)\end{array}$ & \begin{tabular}{l|l}
0.41 & \\
0.007 & \\
0.68 & \\
0.74 \\
0.17 \\
0.42
\end{tabular} \\
\hline $\begin{array}{l}\text { Lumbar BMD, } \\
\text { mean (SD) } \\
\text { Normal, \% } \\
\text { Osteopenia, \% } \\
\text { Osteoporosis, } \\
\%\end{array}$ & $\begin{array}{l}572(27) \\
923(43) \\
657(31)\end{array}$ & $\begin{array}{l}-1.14(1.5) \\
591(43) \\
557(41) \\
216(16)\end{array}$ & $\begin{array}{c}0.0001 \\
0.45 \\
0.0001\end{array}$ & $\begin{array}{l}230(57) \\
132(33) \\
40(10)\end{array}$ & $\begin{array}{c}0.0001 \\
0.03 \\
0.005\end{array}$ & $\begin{array}{c}-0.25(1.5) \\
116(68) \\
47(27) \\
8(5)\end{array}$ & $\begin{array}{c}0.0001 \\
\\
0.0001 \\
0.03 \\
0.11\end{array}$ \\
\hline $\begin{array}{l}\text { Femoral neck } \\
\text { BMD, mean } \\
\text { (SD) } \\
\text { Normal, } n \text { (\%) } \\
\text { Osteopenia, n }\end{array}$ & $\begin{array}{c}586(27) \\
1218\end{array}$ & $\begin{array}{l}617(45) \\
674(49)\end{array}$ & $\begin{array}{l}0.0001 \\
0.001\end{array}$ & $\begin{array}{l}195(47) \\
187(48)\end{array}$ & $\begin{array}{c}0.0001 \\
0.02\end{array}$ & $\begin{array}{l}104(60) \\
58(33)\end{array}$ & $\begin{array}{l}0.0001 \\
0.0001\end{array}$ \\
\hline
\end{tabular}

Acknowledgement: The study was supported with a research grant from the Association for Research in Rheumatology of Marina Baixa (AIREMB)Disclosure of Interests: Jose Rosas Consultant for: Abbvie, Amgen, Bristol, Janssen, Lilly, Merck Sharp \& Dohme, Pfizer, UCB Pharma, Speakers bureau: Abbvie, Amgen, Bristol, Janssen, Lilly, Merck Sharp \& Dohme, Pfizer, UCB Pharma, Catalina Cano: None declared, Ana Pons: None declared, Estíbaliz Ivars: None declared, José Miguel Senabre-Gallego: None declared, Gregorio Santos Soler: None declared, Esteban Salas-Heredia: None declared, José Antonio Bernal-Vidal: None declared, José Alberto García-Gómez: None declared, Xavier Barber: None declared

DOI: 10.1136/annrheumdis-2019-eular.5103

\section{AB0852 DOES INADEQUATE RESPONSE TO DENOSUMAB TREATMENT EXIST?}

Clara Sanguesa, Susana Holgado Pérez, Melania Martínez-Morillo, Jordi CaminsFàbregas, Ivette Casafont-Solé, Annika Nack, Águeda Prior-Español, Maria Aparicio Espinar, Anahy Brandy-Garcia, Lourdes Mateo, Anne Riveros, Alejandro Olive, Laia Gifre. Hospital Germans Trias i Pujol, Badalona, Spain

Background: Denosumab (Dmab), an anti-receptor activator of nuclear factor kappa-B ligand (RANKL) monoclonal antibody, has been shown to increase bone mineral density (BMD) at lumbar spine and proximal femur up to $21.6 \%$ and $9.1 \%$ respectively at 10 years of treatment. Additionally, Dmab has shown a marked decrease of vertebral, nonvertebral and femoral fractures during treatment. Nowadays, the existence of inadequate response to Dmab treatment remains unknown.

Objectives: to describe the clinical, analytical and densitometric characteristics of patients with an inadequate response (IR) to Dmab treatment. IR was defined as the presence of a new fragility fracture during Dmab treatment or a significant decrease in BMD $(\geq 5 \%$ at lumbar spine or $\geq 4 \%$ at proximal femur) within at least 12 months of therapy.

Methods: retrospective study including patients with osteoporosis with an IR to Dmab. Therapeutic compliance was checked by clinical anamnesis and the electronic prescription. Risk factors for osteoporosis, history of fragility fractures, previous anti-osteoporotic treatment, densitometric and analytical data were collected before and at the moment when IR was diagnosed.

Results: Fourteen patients were included (12 women and 2 men) with mean age of $75 \pm 9$ years. The causes of osteoporosis were: postmenopausal $(n=8,57.14 \%)$, induced by glucocorticoids $(n=3,21.43 \%)$, alcoholic ( $n=1,7.14 \%$ ) and multifactorial $(n=2 ; 14.28 \%)$. Nine patients $(64.28 \%)$ had been previously treated with oral or intravenous bisphosphonates fo a mean of $5.8 \pm 2.76$ years. Nine patients $(64.28 \%$ ) had previous vertebral fractures (median 2, range 1-8), 2 of them had also presented a femoral fracture. During Dmab treatment, 7 patients $(50 \%)$ presented a decrease in BMD (mean loss: proximal femur -3.5\%, $p=0.09$; lumbar spine - 5.8\%, $\mathrm{p}=0.046 ;$ ) and 7 had incidental fractures: 5 vertebra (median 1, range 1-4), 1 humerus and 1 femur. The duration of treatment with Dmab was $3.82 \pm 1.85$ years in patients who sustained fragility fractures and $2.39 \pm 1.4$ years in patients with a BMD decrease. A multiple myeloma was diagnosed in a patient with vertebral fractures dur ing Dmab treatment. After The identification of Dmab Rl most patients mantained same treatment. Of the patients with incidental fragility frac tures, 2 started combined treatment with teriparatide and Dmab, 1 changed to teriparatide and 2 maintained the same treatment. Of the 7 patients with BMD only 1 changed to zoledronic acid.

Conclusion: Most patients with IR to Dmab treatment had previous fragility fractures and had been previously treated with bisphosphonates for a mean duration of 5 years. The patients with a significant decrease in BMD had lesser duration of Dmab treatment than those who sustained fractures during Dmab treatment. Only one patient had a clinical cause for the IR development.

Disclosure of Interests: None declared

DOI: 10.1136/annrheumdis-2019-eular.7913

\section{AB0853 BONE DENSITOMETRY IN CROHN'S DISEASE: RISK FACTORS FOR OSTEOPENIA AND OSTEOPOROSIS}

Sabrine Soua ${ }^{1}$, Dhilel Issaoui ${ }^{2}$, Hela Kchir ${ }^{2}$, Nouha Trad ${ }^{1}$, Hatem Chaabouni ${ }^{1}$, Nadia Maamouri ${ }^{1}{ }^{1}$ La Rabta Hospital, Tunis, Tunisia; ' La Rabta Hospital, Tunis, Tunisia

Background: Osteoporosis and osteopenia are common situations in chronic inflammatory bowel disease (IBD), particularly in crohn's disease. 